Abstract P213 Table 1 Feedback on Ml sessions (33 interventions)

\begin{tabular}{lll}
\hline & $\begin{array}{l}\text { Strongly } \\
\text { Agree/Agree }\end{array}$ & Not Agree \\
\hline "My knowledge on SH has improved" & $80 \%$ & $20 \%$ \\
"My confidence to look after my SH has improved" & $80 \%$ & $20 \%$ \\
"My motivation to look after my SH has improved" & $93 \%$ & $7 \%$ \\
"My knowledge on the different ways I can test for & $69 \%$ & $31 \%$ \\
HIV/STIs has improved" & & \\
"My knowledge of PEP/where to access has improved" & $54 \%$ & $46 \%$ \\
"My confidence in managing or abstaining from & $93 \%$ & $7 \%$ \\
drugs/alcohol; making better choices with regard to & & \\
my SH has improved" & & \\
\hline
\end{tabular}

Conclusions Unprotected sex is common among MSW. Early MI results show good improvement in knowledge and risk taking behaviour. High levels of drug/alcohol use and self-harm require close links to mental health services. Pro(TECT) is unique in accessing this 'hard-to-reach' population and offers a holistic service of harm reduction.

\section{P214 ARE WE MISSING SOMETHING? EXTRA-GENITAL CT/GC NAAT TESTING IN FEMALE PATIENTS ATTENDING A YOUNG PERSONS CLINIC}

Elizabeth Williams, Sarah Ramsay*, Sarah Creighton, Tristan Barber. Homerton University Hospital, London, UK

\subsection{6/sextrans-2015-052126.258}

Background/introduction Our service has a dedicated Young Persons Clinic (YPC) for women age $\leq 25$. Current policy is to only offer routine vulvo-vaginal (VVS) or cervical CT/GC NAAT swabs for female patients but we are aware that STIs in non-genital sites may therefore be missed. From 15/04/14 we offered female patients attending our YPC VVS/cervical and extragenital (throat and rectum) swabs, regardless of exposure stated.

Aim(s)/objectives To quantify the number and result of CT/GC extragenital samples from YPC female patients.

Methods NAAT results for all women attending YPC between $15 / 04 / 14-16 / 09 / 14$ were extracted retrospectively from an electronic database held within the clinic.

Results

\begin{tabular}{lll}
\hline & $\begin{array}{l}\text { Number of } \\
\text { patients }\end{array}$ & $\%$ \\
\hline STI Screens & 193 & \\
Acute STI diagnosed at that visit (including TV and PID) & $29 / 193$ & 15 \\
Positive CT/GC NAAT at that visit & $24 / 193$ & 12 \\
More than one site sampled & $34 / 193$ & 18 \\
with positive extragenital CT/GC NAAT *and negative & $4 / 34$ & 12 \\
VVS/cervical CT/GC NAAT & & \\
\hline *GC throat $\times 2$, CT throat $\times 1$, CT throat + GC rectal $\times 1$ & &
\end{tabular}

42 patients were documented to have been offered extragenital swabs. Of those, 34 (81\%) accepted.

Discussion/conclusion Uptake of extragenital site testing was low. This is likely to reflect low rates of offering extragenital swabs, as there was a high rate $(81 \%)$ of acceptance where an offer was documented. Five infections were solely identified from extragenital testing. It is recognised that a positive result does not necessarily imply infection and extragenital tests are currently unlicensed. Therefore this data suggests that further review would be useful.

\section{P215 DELIVERING STI SERVICES IN HOSTELS FOR HOMELESS INDIVIDUALS}

Elizabeth Williams, Sarah Macauley, Sarah Ramsay*, Sarah Creighton. Homerton University Hospital, London, UK

\subsection{6/sextrans-2015-052126.259}

Background/introduction Residents of hostels for homeless individuals have a disproportionate burden of mental and physical health needs, which can expose them to risk of blood born viruses (BBVs) and STIs. Our borough runs 5 hostels which address health and social needs as well as provide accommodation.

Aim(s)/objectives To report on a pilot aiming to improve diagnosis and treatment of BBVs and STIs of residents of these 5 hostels.

Methods Between 14/02/2012 and 14/02/2013 five hostels were visited a minimum of two times. CT/GC NAATs and HIV, Syphilis, Hepatitis B and C serology were offered as well as signposting to other services.

Results

\begin{tabular}{ll}
\hline Number of residents: & \\
- Seen & 56 \\
- With past/current IVDU & $36 / 56(64 \%)$ \\
- With known HIV & $3 / 56(5 \%)$ \\
- With known HCV & $6 / 56(10 \%)$ \\
- Who had previously tested for HIV & $41 / 56(73 \%)$ \\
- Who had tested for HIV in the preceding year & $29 / 56(52 \%)$ \\
- Who had BBV serology on visit & $21 / 56(38 \%)$ \\
- Who had BBV serology on visit that had not previously tested & $8 / 56(14 \%)$ \\
- Who had CT/GC testing on visit & $54 / 56(96 \%)$ \\
New positive diagnoses & $1 / 56(2 \%)-$ HCV \\
\hline
\end{tabular}

Discussion/conclusion Half the residents had been tested for HIV in the preceding year. $14 \%$ had never previously tested for BBV. 38\% accepted BBV testing at this service and 96\% accepted $\mathrm{CT} / \mathrm{GC}$ testing. One new infection was diagnosed. This suggests that existing services meet the needs of the majority of this group. However, this additional service provided support to a minority of individuals who had been unable to negotiate existing services.

\section{P216 MONITORING GENDER RATIO OF GASTROINTESTINAL INFECTION LABORATORY REPORTS AS A MECHANISM FOR IDENTIFYING POSSIBLE INCREASES AMONG MEN WHO HAVE SEX WITH MEN, ENGLAND, 2003-2013}

${ }^{1}$ Piers Mook*, 'Sanch Kanagarajah, 'Daniel Gardiner, ${ }^{1,2}$ Marko Kerac, 'Gwenda Hughes, ${ }^{1,3}$ Nigel Field, ${ }^{1,4}$ Noel McCarthy, ${ }^{1}$ lan Simms, ${ }^{1}$ Chris Lane, ${ }^{1}$ Bob Adak, ${ }^{1}$ Paul Crook. ${ }^{1}$ Public Health England, London, UK; ${ }^{2}$ London School of Hygiene and Tropical Medicine, London, UK; ${ }^{3}$ University College London, London, UK; ${ }^{4}$ University of Warwick, London, UK

\subsection{6/sextrans-2015-052126.260}

Background Since 2011, an increase in Shigella flexneri has been observed in men due to faecal-oral transmission associated with sexual contact between men who have sex with men (MSM). Sexual history is not routinely collected for cases of gastrointestinal infections. 
Aims To use gender ratio to detect greater than expected numbers of gastrointestinal infections in MSM.

Methods We examined annual male to female ratios of laboratory confirmed patient-episodes from those aged 16-65 years with no known history of travel for eight gastrointestinal pathogens (Campylobacter, Cryptosporidium, Giardia, Hepatitis A, Norovirus, Salmonella, Shigella, and VTEC) in England between 2003 and 2013. Chi-squared tests for linear trend were conducted and a male to female ratio of more than two was considered suggestive of an excess. Sub-analyses by age and high-risk areas (London, Brighton and Manchester) were conducted.

Results An increased linear trend and excess of male episodes was observed for Shigella ( $\mathrm{p}<0.001$; m:f ratio of 2.0 and 2.5 in 2012 and 2013, respectively) but not the other gastrointestinal infections. Consistent with MSM-mediated transmission, the excess of male Shigella episodes was most pronounced among those aged 25-49 years (ratios of 2.4 and 2.9) and those in high-risk areas (ratios of 2.9 and 4.0); no excess was observed among children.

Conclusion This method identified the recent outbreak of Shigella and routine application might alert public health authorities to some future gastrointestinal infection outbreaks in MSM. Utility of this approach to detect excess episodes among MSM is likely to be pathogen specific and dependent on several factors including R0.

\section{P217 HIGH DEMAND FOR AN MSM CLINIC PILOT IN A DISTRICT GENERAL HOSPITAL}

${ }^{1,2}$ Mamatha Oduru*, ${ }^{2}$ Emma Wainwright, ${ }^{2}$ Janice Burnett, ${ }^{2}$ Alan Tang. ${ }^{1}$ The Garden Clinic, Slough, UK; ${ }^{2}$ The Florey Unit, Reading, UK

10.1136/sextrans-2015-052126.261

Background/introduction A tailored sexual health service for men who have sex with men (MSM) was piloted in a district general hospital. This was in response to a 'Gay Pride' survey in which $80 \%$ of surveyed attendees requested a specialised local service.

Aim(s)/objectives This clinic explored the feasibility and acceptability of a targeted MSM service in the district general hospital setting.

Methods The pilot had 9 clinics over 3 months. Each patient was fast-tracked to a multidisciplinary team (doctor, nurse and health advisor). A rapid HIV test (result within $24 \mathrm{~h}$ ) was offered to every patient as point of care testing was unavailable in the service. Data and patient feedback were analysed in SPSS version 22.

Results A total of 13 patients attended the clinic (new, $\mathrm{N}=12$, follow up, $\mathrm{N}=1$ ). Age range was 22 to 67 years old (mean 33.7, standard deviation 13.27) and all were Caucasian. 6/13 attended for an asymptomatic screen; the prevalence of sexually transmitted infections (STI's) was 46\%. All tested HIV negative. $61.5 \%$ had no prior immunity to Hepatitis B. $92.3 \%$ considered the rapid HIV test an incentive to attend and 100\% wanted a future point of care test. There was favourable patient feedback.

Discussion/conclusion The clinic pilot was positively received and the majority rated it preferable to the routine genitourinary clinic. There was a high prevalence of STI's and high rate of opportunistic Hepatitis B vaccination. There is a notable demand for rapid HIV testing. Our tailored MSM clinic encouraged attendance in a high risk provincial population.
P218 RISING STI RATE IN FEMALE SEX WORKERS ATTENDING AN INNER CITY DEDICATED CLINIC

${ }^{1}$ Elizabeth Williams, ${ }^{2}$ Kim Leverett*, ${ }^{1}$ Vanessa Apea. ${ }^{1}$ Ambrose King Centre, Barts Health NHS Trust, London, UK; ${ }^{2}$ Homerton University Hospital NHS Trust, London, UK

\subsection{6/sextrans-2015-052126.262}

Background/introduction Our GUM service has a dedicated sex worker (SW) clinic. In 2014 there was an anecdotal increase in in CT and GC diagnoses in patients attending this clinic therefore a retrospective case note review was performed to assess this observation and explore causal factors.

Aim(s)/objectives To compare number of CT/GC infections in the SW clinic in 2012 and 2014.

Methods Notes were reviewed for age, ethnicity and CT/GC codes in all patients seen in 2012 and 2014. The notes of those found to be CT or GC positive had a more detailed review for type of services offered, condom use, place of work (i.e. flat, sauna). Chi-squared test was used to calculate $\mathrm{p}$ value.

Results

\begin{tabular}{llll} 
Abstract P218 Table 1 & \multicolumn{4}{l}{ STI rates in female sex workers } & \\
\hline Year & 2012 & 2014 & P value \\
\hline Number of patients: & 192 & 140 & \\
- Attending & $19-58$ & $19-54$ & \\
- Age Range & $5 / 192(2.6 \%)$ & $19 / 140(13.6 \%)$ & 0.0002 \\
- With either CT or GC at least once & 0 & $3 / 140(2.1 \%)$ & \\
- With both CT and GC concurrently & 0 & $3 / 140(2.1 \%)$ & \\
- That had repeat infections in the same year & 0 & 16 & \\
Total number of CT infections & 4 & 11 & \\
Total number of GC infections & 1 & &
\end{tabular}

Discussion/conclusion There was a significant increase in STIs from 2012 to 2014. In addition, concurrent CT and GC infection and repeat infections were seen in 2014, but not in 2012. This observation has guided an update in patient education materials and a more targeted approach to outreach. Possible infection clusters are being explored.

\section{P219 DOES A SPECIALISED MSM CLINIC IN A DISTRICT GENERAL HOSPITAL ATTRACT A RISKIER POPULATION?}

1,2 Mamatha Oduru ${ }^{*},{ }^{2}$ Emma Wainwright, ${ }^{2}$ Janice Burnett, ${ }^{2}$ Alan Tang. ${ }^{1}$ The Garden Clinic, Slough, UK; ${ }^{2}$ The Florey Unit, Reading, UK

10.1136/sextrans-2015-052126.263

Background/introduction A sexual health service for men who have sex with men (MSM) was piloted due to local demand for a specialised clinic with an understanding of MSM risk taking behaviour and sexual practices.

Aim(s)/objectives To explore if an MSM clinic in a district general hospital attracts a patient population with riskier sexual behaviour compared to the normal genitourinary (GU) service. Methods Risk data was collected from all MSM patients attending the specialised and routine GU clinics over a 3 month period using self-completed questionnaires. 'High risk' behaviour was classified as any of the following within the past 3 months: group sex, sex parties, chem-sex, casual partner sourcing online, sexual activity in London and number of partners. Data were analysed in SPSS version 22.

Results Total sample size was 40 (MSM clinic, $\mathrm{N}=13$, GU clinic, $\mathrm{N}=27$ ). Age range was 18 to 67 years old (mean 32.47, 